\title{
Integral Representations \\ of Axially Symmetric Potential Functions
}

\author{
Albert E. Heins \& Richard C. MacCamy \\ Communicated by A. ERDÉLYI
}

\section{Introduction}

This paper is concerned with solutions of elliptic partial differential equations in three variables when there is an axis of symmetry. The simplest example is Laplace's equation in $x, y$, and $z$. Solutions which are symmetric about the $z$-axis are then functions $u(r, z)$ of $z$ and $r^{2}=x^{2}+y^{2}$, satisfying

$$
L_{k}[u]=u_{r r}+k r^{-1} u_{r}+u_{z x}=0, \quad k=1 .
$$

It is well known that solutions of (1.1) which are regular in a region $R$ of $r>0$, having as part of its boundary a segment $\lambda$ of the $z$-axis, are uniquely determined in all of $R$ by their values on $\lambda$ if those values are real analytic. It can be shown that the function $u(0, z)$ is analytic for complex $\zeta=z+i \xi$ in $R$, and the uniquely determined $u(r, z)$ is given by the so-called "Laplace integral",

$$
u(r, z)=\frac{1}{\pi} \int_{0}^{\pi} u(0, z+i r \cos \vartheta) d \vartheta
$$

Various extensions of the above ideas suggest themselves. A harmonic function in $(n+2)$ variables $z, x_{1}, \ldots, x_{n+1}$, symmetric about the $z$-axis is a function $u(r, z)$ which satisfies equation $(1,1)$ when $k=n$ and $r^{2}=x_{1}^{2}+\cdots+x_{n+1}^{2}$. An equation of a similar form arises if we decompose a three dimensional harmonic function $\Omega(x, y, z) \doteq \omega(r, \vartheta, z)$ with $r^{2}=x^{2}+y^{2}$ and $\vartheta=\arctan y / x$ into a Fourier series

$$
\omega(r, \vartheta, z)=\sum_{n}\left[a_{n}(r) \cos n \vartheta+b_{n}(r) \sin n \vartheta\right] .
$$

In this case the functions $r^{n} a$, and $r^{n} b_{n}$ satisfy equation (1.1) with $k=2 n-1$. WeINSTLin in a series of papers has studied equation (1.1) for an arbitrary $k$; see, for example, WEINSTEIn [1] as well as the work of ERDÉLYI [2].

The particular extension we wish to discuss in detail is due to HENRICI [3], $[4],[5]$. Let. $e[u]$ denote the differential operator

$$
e[u]=a(z) u_{z}+b(z) u .
$$

HeNRICI has studied the equation

$$
L_{k}[u]+e[u]=0
$$


when $a$ and $b$ are analytic functions of a complex variable $z$ in some region $R$ containing a portion $\lambda$ of the real $z$-axis and are real on $\lambda$. He showed by a power series argument [3] that for $k>0$ any solution $u(r, z)$ of (1.3), which is analytic on $r=0$, is uniquely determined by its values on $r=0$. The following interesting observation was also made by him. Let $u_{0}(r, z)$ be a solution of the equation

$$
L_{0}\left[u_{0}\right]+e\left[u_{0}\right]=0
$$

such that

The integral

$$
u_{0}(0, z)=f(z)
$$

$$
P(r, z ; f)=C_{k} \int_{0}^{\pi} u_{0}(r \cos \vartheta, z) \sin ^{k-1} \vartheta d \vartheta
$$

where $C_{k}^{-1}=\int_{0}^{\pi} \sin ^{k-1} \vartheta d \vartheta$ and $k>0$ is a solution of equation (1.3) such that $P(0, z ; f)=f(z)$.

Having established (1.5), HENRICI proceeds by the operator method of BeRgMan [8]. The analyticity of $a$ and $b$ imply that the function $u_{0}(r, z)$ is analytic, and hence one may permit $r$ and $z$ to be complex. Upon introducing the new variables $\zeta=z+i r$, and $\zeta^{*}=z-i r$, equation (1.4) is converted into a formally hyperbolic equation. The method of RIEMANN is now applicable and yields an integral representation for $u_{0}$ in terms of $f$. When this representation is substituted into (1.5), one obtains a second representation of the form

$$
P(r, z ; f)=\int_{0}^{\pi} K(r, z, \vartheta) f(z+i r \cos \vartheta) d \vartheta
$$

a generalization of (1.2). It is shown that all solutions of (1.3) which are analytic on a portion of the $z$-axis have such a representation with $f(\zeta)=u(0, \zeta)$.

HENRICI used formula (1.6) to transfer expansion theorems for analytic. functions of a complex variable to solutions of (1.4), thus generating complete sets of solutions. The interest of the authors was prompted by a desire to discuss boundary-value problems for equations of the type (1.3) by translating them into information about the associated analytic functions. The inversion formulas which give the function $f$ in (1.6) in terms of $P$ are then of some interest. Some success has been' achieved in this program in the special case $e[u]=\mu u, \mu$ a constant, [6] and [7].

The present paper presents our contributions to the general theory. We remove the restriction that the coefficients in $e[u]$ depend only on $z$. In order to minimize the computations, however, we limit ourselves to the equation

$$
L_{1}[u]+q(r, z) u=0,
$$

where $q$ is an entire function of $r^{2}$ and $z$. In the next section we shall give a proof of the uniqueness theorem which will illustrate once again the intimate connection between elliptic partial differential equations with analytic coefficients and hyperbolic partial differential equations. Section 3 is mainly devoted to obtaining an analog of the representation (1.6). This is accomplished by constructing a convergent successive approximation procedure from which we can 
construct the kernel in (1.6). In addition to this, we discuss some useful properties of this kernel. Following this, we discuss the connection between the regularity of the solution of equation (1.7) and the analyticity of the boundary data at $r=0$ of this solution. We conclude with the case for which $q$ is independent of $z$, putting special emphasis on a discussion of several special inversion formulas.

\section{The Uniqueness Theorem}

We shall define a semi-region $R_{1}$ to be an open, simply-connected set of the $r-z$ plane, lying in $r>0$ and such that its boundary contains a single segment $A$ of the axis. The closed interval $\lambda: z_{1} \leqq z \leqq z_{2}$ will be called an interior segment of $A$ if for some $\alpha>0$ the rectangle $W_{\alpha}: z_{1} \leqq z \leqq z_{2}, 0<r<\alpha$ lies entirely in $R_{1}$. Let us consider the equation

$$
L(u)=u_{r r}+r^{-1} u_{r}+u_{z z}+q u=0
$$

where $q(r, z)$ is an entire function of $r^{2}$ and $z$. A solution of (2.1) which is of class $C^{2}$ in the closure $\bar{R}$ of $R_{1}$ will be called a regular symmetric function (RSF) in $R$.

We remark that the continuity of $u_{r r}$ on $\Lambda$ demands that $u_{r}$ should vanish identically on $A$. It follows that a RSF can be continued as an even function of $r$ to the image of $R_{1}$ with respect to the $z$-axis. Thus, there is no loss in generality if we consider a RSF in a region $R$ consisting of a semi-region $R_{1}$, its image with respect to the $z$ axis and the segment $\Lambda$.

Theorem 1. Let $R$ be a region and $\lambda$ an interior segment $r=0, z_{1} \leqq z \leqq z_{2}$. Suppose that $g(z)$ is a real analytic function of $z$ on $\lambda$. Then there exists at most one RSF $u(r, z)$ in $R$ such that $u(0, z)=g(z)$ on $\lambda$.

The first observation we wish to make is that a RSF $u(r, z)$ is a real analytic function of $r$ and $z$ in $R$. In the semi-region $R_{1}$, and its image this is obvious since $u$ is a solution of an elliptic differential equation with analytic coefficients. In order, to see that $u$ is also analytic on $\lambda$.we observe that the function $u_{1}(x, y, z)=$ $u\left[\left(x^{2}+y^{\frac{1}{2}}\right)^{\frac{1}{2}}, z\right]$ is an axially-symmetric function in $x, y$ and $z$ in the cylinder $C$ obtained by rotating the closure of the rectangle $W_{\alpha}$ about the $z$ axis. Moreover since $u$ is a function of $r^{2}$ and is of class $C^{2}$ in $r$, we see that $u_{1}$ is of class $C^{2}$ in $C$ and hence must be analytic. Thus if $z_{0} \in \lambda$, we have

$$
u_{1}(x, y, z)=\sum_{m} \sum_{n} \sum_{p} A_{m n p} x^{m} y^{n}\left(z-z_{0}\right)^{p} .
$$

Upon putting $y=0$, so that $x=r$, we have

$$
u(r, z)=\sum_{m} \sum_{\gtrless} A_{m 0 p} x^{m}\left(z-z_{0}\right)^{p} .
$$

In view of the analyticity of $u$, the theorem will be proved if we can show that $u$ is identically zero in any region containing $\lambda$, if $g(z)$ vanishes on $\lambda$. Since $u$ is analytic in $W_{\alpha}$, we may continue $u(r, z)$ as an analytic tunction into some region $D$ for complex $z$ while we retain $r$ real. The extent of the region $D$ is of no interest here save that it should contain $W_{\alpha}$. 
Let us denote the continued function by $\hat{u}(r, z)$. By the principle of permanence of a functional equation we conclude that equation (2.1) still holds. We write $z=z^{\prime}+i z^{\prime \prime}$, and for a fixed $z^{\prime}=\beta$ in $\lambda$ we write $\hat{u}\left(r, \beta+i z^{\prime \prime}\right)=U\left(r, z^{\prime \prime}\right)$. In view of the equation (2.1), $U$ satisfies the hyperbolic equation

$$
U_{r,}+r^{-1} U_{r}-U_{z^{\prime \prime} z^{\prime \prime}}+Q\left(r, z^{\prime \prime}\right) U=0,
$$

in $D$ for $z^{\prime}=\beta$ where now $Q\left(r, z^{\prime \prime}\right)=q\left(r, \beta+i z^{\prime \prime}\right)$. Let $D^{\prime}$ be that subset of $D$ obtained by letting $r=0$ and $z^{\prime}=\beta$ and which therefore intersects $\lambda$. Since $u$ is a $\mathrm{RSF}$ we have that $u_{r}(0, z) \equiv 0$ on $\lambda$. Hence by continuation $\hat{u}_{r}(0, z) \equiv 0$ in $D^{\prime}$, and therefore

$$
U_{r}\left(0, z^{\prime \prime}\right) \equiv 0 \quad \text { on } \gamma
$$

where $\gamma$ is the intersection of $D^{\prime}$ and $z^{\prime}=\beta$. Similarly, the vanishing of $u(0,2)$ on $\lambda$ leads to

$$
U\left(0, z^{\prime \prime}\right) \equiv 0 \quad \text { on } \gamma \text {. }
$$

Since the segment $\gamma$ forms a part of the boundary of $D$ at $z^{\prime}=\beta$, we see that equations (2.2) - (2.4) provide an initial-value problem for a hyperbolic equation in two variables $r$ and $z^{\prime \prime}$. It differs from the usual problem only in that the data are given on a singular line. This problem was considered in [9] where it was shown that the function $U$ must vanish in some neighborhood of $r=0$. Since this is true for each fixed $z^{\prime}$ in $\lambda$, it follows that $u$ vanishes in some neighborhood of $r=0$. Hence upon specializing to $z^{\prime \prime}=0, u$ vanishes near $r=0$, and the proof is complete.

\section{Laplace İntegrals}

The uniqueness theorem we have just proved now suggests an existence theorem. As we shall show in the next section, however, such a theorem will be false without further restriction on $g(z)$. The appropriate existence theorem reads as follows.

Theorem 2. Let $g(\zeta)$ be an analytic function of the complex variable $\zeta=z+i r$ in a region $R$ with $g$ real on $\Lambda$. Then there exists a $R S F u(r, z)$ in $R$ such that $u(0, z)=g(z)$ on $A$.

We shall prove this theorem by obtaining an analog of the Laplace integral (1.2). We shall show that for each $q$ which is an entire function of $r^{2}$ and $z$, there exists a function $E(r, z, t)$ which is analytic in $r$ and $z$ such that the RSF of Theorem 2 has the representation

$$
u(r, z)=\int_{z-i r}^{z+i r} E(r, z, t) g(t) d t .
$$

Two observations may be noted. One is that $E$ does not depend on the region $R$, and the second is that the representation (3.1) for equation (1.1) reduces to (1.2).

Before we establish the representation (3.1) it is instructive to indicate why it shoukd be expected to hold. It has already been indicated that the solutions of (2.1) are analytic functions of the complex variables $r$ and $z$. In order to make this remark more useful, let us introduce, following [5], the complex 
variables $\zeta=z+i r$ and $\zeta^{*}=z-i r$. Real $r, z$ space is then characterized by $\bar{\zeta}^{*}=\zeta$. If we again denote by $\hat{u}(r, z)$ the continuation of $u(r, z)$ to complex $r$ and $z$ and put

and

$$
\Phi\left(\zeta, \zeta^{*}\right)=u\left[\left(\zeta-\zeta^{*}\right) / 2 i,\left(\zeta+\zeta^{*}\right) / 2\right]
$$

$$
Q\left(\zeta, \zeta^{*}\right)=q\left[\left(\zeta-\zeta^{*}\right) / 2 i,\left(\zeta+\zeta^{*}\right) / 2\right],
$$

we find that equation (2.1) becomes

$$
\Phi_{\zeta \zeta^{*}}+\frac{1}{2\left(\zeta-\zeta^{*}\right)}\left[\Phi_{\zeta^{*}}-\Phi_{\zeta}\right]+\frac{Q \Phi}{4}=0
$$

It is known that $u(0, z)=g(z)$ and $u_{r}(0, z) \equiv 0$ on some segment of $r=0$. In the complex $r, z$ space, $r=0$ corresponds to $\zeta=\zeta^{*}$. Hence, in terms of $\Phi$, these conditions yield

$$
\Phi=g(\zeta)
$$

and

on $\zeta^{*}=\zeta$.

$$
\Phi_{\zeta}=\Phi_{\zeta *}
$$

Equations (3.2)-(3.4) show that $\Phi$ is, at least formally, the solution of a non-characteristic initial-value problem for a hyperbolic equation, the data being given on a singular line. Since the equation is linear, it may be expected that the method of Riemann will be applicable. Let the function $\Psi\left(\zeta, \zeta^{*}, w, w^{*}\right)$ satisfy the following hyperbolic equation and auxiliary conditions:

and

$$
\begin{aligned}
\Psi_{\zeta \zeta^{*}}+\frac{1}{2\left(\zeta-\zeta^{*}\right)}\left[\Psi_{\zeta}-\Psi_{\zeta^{*}}\right]+\left[\frac{Q}{4}-\frac{1}{\left(\zeta-\zeta^{*}\right)^{2}}\right] \Psi=0 \\
\frac{1}{2\left(w-\zeta^{*}\right)} \Psi\left(w, \zeta^{*}, w, w^{*}\right)+\Psi_{\zeta^{*}}\left(w^{*}, \zeta^{*}, w^{*}\right)=0 \\
\frac{1}{2\left(\zeta-w^{*}\right)} \Psi\left(\zeta, w^{*}, w^{*}\right)-\Psi_{\zeta}\left(\zeta, w^{*}, w, w^{*}\right)=0
\end{aligned}
$$

$$
\Psi\left(w, w^{*}, w, w^{*}\right)=1
$$

where equation (3.5) is the adjoint of equation (3.2). The existence of $\Psi$ may be established by observing that $\Psi$ is a solution of equation (3.2) in the variables $w$ and $w^{*}$. The equations (3.6)-(3.8) may thus be considered to provide conditions for a characteristic initial value problem for equation (3.2), the solution of which can be established by the method of successive approximations.

If we now assume the existence of $\Psi$, it may be used in the standard manner to construct the solution of equation (3.2) which will satisfy (3.3) and (3.4). There is, however, one essential difference which reflects the singular nature of the problems. From (3.6) $-(3.8)$ we find that

and

$$
\Psi\left(w, \zeta^{*}, w, w^{*}\right)=\left\{\frac{w-\zeta^{*}}{w-w^{*}}\right\}^{\frac{1}{2}}
$$

$$
\Psi\left(\zeta, w^{*}, w, w^{*}\right)=\left\{\frac{\zeta-w^{*}}{w-w^{*}}\right\}^{*}
$$


It follows that

$$
\Psi\left(w, w, w, w^{*}\right)=\Psi\left(w^{*}, w^{*}, w, w^{*}\right)=0 .
$$

Hence the customary boundary terms in the Riemann contour representation vanish, and we obtain for the solution of the non-characteristic problem (3.2) to (3.4) an expression of the form

$$
\Phi\left(w, w^{*}\right)=\int_{w}^{w^{*}} K\left(w, w^{*}, \zeta\right) g(\zeta) d \zeta
$$

where $K$ is a combination of $\Psi$ and its derivatives. If we now put $w^{*}=\bar{w}$, the representation (3.9) will yield an expression of the form of (3.1).

The determination of the Riemann function $\Psi$ would be, in general, a formidable task. Fortunately, all that is needed is the particular combination $K$. For orientation let us consider the case $q=0$. In this case the function $\Psi$ has been given by Darboux [10] in terms of the hypergeometric function, and (3.9) becomes $[6]$

$$
\Phi\left(w, w^{*}\right)=\frac{i}{\pi} \int_{w}^{w^{*}} g(\zeta)\left[(w-\zeta)\left(w^{*}-\zeta\right)\right]^{-\frac{1}{2}} d \zeta
$$

Hence for (3.1) we obtain

$$
u(r, z)=-\frac{i}{\pi} \int g(\zeta)\left[(z-\zeta)^{2}+r^{2}\right]^{-\frac{1}{2}} d \zeta
$$

Suppose now that the line segment $\zeta=z+i \tau,-r \leqq \tau \leqq r$ is contained in the domain of regularity of $g(\zeta)$. The substitution $\zeta=z+i r \cos \vartheta$ will convert the representation (3.11) into the Laplace integral (1.2).

We could make precise the steps we have indicated in arriving at (3.1), but it is possible simply to verify the final result. We write $\Gamma=(z-\zeta)^{2}+r^{2}$ and let $H(r, z, \zeta)$ be an entire function of the complex variable $\zeta$ for all real $r$ and $z$. Let $\mathfrak{\Im}$ be the class' of functions $h$ which are analytic in the region $R$, which we have taken to be symmetric with respect to the real $z$ axis. We intruduce linear transformations $\Phi^{(n)}$ over $\mathfrak{\Im}$ by

$$
\Phi^{(n)}(r, z ; H ; h)=-\frac{i}{\pi} \int_{z=-i r}^{z+i r} H(r, z, \zeta) \Gamma^{n-1} h(\zeta) d \zeta
$$

where $(r, z) \in R$ and $\zeta \in \varkappa$ where $x$ is a curve joining $z-i r$ to $z+i r$ and lying entirely in $R$. We may summarize the properties of $\Phi^{(n)}$ as follows.

Lemma 1. (i) The value of $\Phi^{(n)}$ is independent of the curve $x$ :

(ii) $\Phi^{(0)}(0, z ; H ; h)=H(0, z z) h(z)$;

(iii) $\Phi^{(n)}(0, z ; H ; h)=0, \quad n \geqq 1$;

and

$$
\Phi_{r}^{(n)}(0, z ; H ; h)=0, \quad n \geqq 1 ;
$$

(iv) $L[\Phi]^{(n)}=-\frac{i}{\pi} \int_{z-i}^{z+i r}\left\{L[H] \Gamma^{n-\frac{1}{2}}+4\left(n-\frac{1}{2}\right) m_{n}[H] \Gamma^{n-1}\right\} h(\zeta) d \zeta$,

$$
n \geqq 1
$$


where

$$
m_{n}[H]=r H_{z}+(z-\zeta) H_{z}+n H .
$$

In (ii) and (iii) the point $(0, z)$ is to be on an interior segment of $R \cap(r=0)$.

To prove (i) we observe that the only singularities of the integral are branch points of order $\frac{1}{2}$ at $\zeta=z \pm i r$. Any two curves joining these points and lying in $R$ will thus enclose a domain on the Riemann surface of $\Gamma^{1}$ within which the integrand will be single-valued. Hence the integrals over the two curves will be the same.

The proof of (ii) and (iii) follows from a transformation of variables which we have already indicated. Since $(0, z)$ is in an interior segment, the line $\zeta=z+i \tau$, $-r \leqq \tau \leqq r$ lies entirely in $R$ provided $r$ is sufficiently small. For such an $r$ we obtain, upon using (i), the value of $\Phi^{(n)}$ by integrating along this line. Upon introducing the variable $\vartheta$ by the substitution $\zeta-z=i r \cos \vartheta,(3.12)$ assumes the form

$$
\text { (3.12a) } \Phi^{(n)}(r, z ; H ; h)=\frac{1}{\pi} \int_{0}^{\pi} H(r, z, z+i r \cos \vartheta) h(z+i r \cos \vartheta) r^{2 n} \sin ^{2 n} \vartheta d \vartheta \text {, }
$$

from which (ii) and (iii) follow immediately.

For $n \geqq 2$, the terms arising from the limits of integration in $\Phi_{r}^{(n)}, \Phi_{r r}^{(n)}$ and $\Phi_{z z}^{(n)}$ vanish because $\Gamma$ is zero at both limits. Thus $L\left[\Phi^{(n)}\right]$ can be computed by differentiating under the integral sign, and establishing (iv) is an elementary computation. For $n=1$, we could carry out the verification from (3.12), although some care would be necessary due to the singular nature of the derivatives of $\Gamma^{1}$ at $\zeta=z \pm i r$. This difficulty may be avoided by using. (3.12a). Finally, the special case $\Phi^{(0)}[r, z ; 1 ; h]$ is a solution of $(1.1)$ with $\Phi^{(0)}(0, z ; 1 ; h)=h(z)$, as we have already indicated. Thus we have

$$
L\left[\Phi^{(0)}(r, z ; 1 ; h)\right]=-\frac{i}{\pi} q(r, z) \int_{z-i r}^{z+i r} h(\zeta) \Gamma^{-\frac{1}{2}} d \zeta .
$$

Lemma 1 now suggests the following construction. Suppose we define the functions $H^{(n)}(r, z, \zeta)$ recursively by the relation

$$
4\left(n-\frac{1}{2}\right) m_{n}\left[H^{(n)}\right]=-L\left[H^{(n-1)}\right], \quad n \geqq 1
$$

and $H^{\prime 0)}=1$. We shall prove the following result.

Lemma 2. There exists a unique set of functions $\left\{H^{(n)}\right\}$ satisfying (3.14) which are regular at $r=0$. If $\zeta=\xi+i \eta$, the series

$$
S(r, z, \zeta)=\sum_{n=0}^{\infty} H^{(n)}(r, z, \zeta) \Gamma^{n}
$$

converges uniformly for $(r, z)$ and $(\xi, \eta)$ in any closed bounded region $R$.

Let us assume the validity of Lemma 2 for the present, so that we may complete the proof of Theorem 2. From the uniform convergence of the series which defines $S$ we can write

$$
\begin{aligned}
\Phi(r, z ; f) & =-\frac{i}{\pi} \int_{z-i r}^{z+i r} S(r, z, \zeta) g(\zeta) I^{-\frac{1}{2}} d \zeta \\
& =\sum_{n=0}^{\infty} \Phi^{(n)}\left[r, z ; H^{(n)} ; g\right]
\end{aligned}
$$


for any function $g(\zeta)$ regular in a region $R$. The integral is taken along any arbitrary curve joining $z-i r$ to $z+i r$ and lying in $R$. From Lemma 1, the recurrence relation (3.14) and (3.13) we have

$$
\begin{aligned}
L[\varphi] & =-\frac{i}{\pi} q(r, z) \int_{z=-i r}^{z+i r} g(\zeta) \Gamma^{-\frac{1}{2}} d \zeta-\sum_{n=1}^{\infty} \frac{i}{\pi} \int_{z=i r}^{z+i r}\left\{L\left[H^{(n)}\right] \Gamma^{n-1}-L\left[H^{(n-1)}\right] \Gamma^{n-1}\right\} g(\zeta) d \zeta \\
& =-\frac{i}{\pi} q(r, z) \int_{z-i r}^{z+i r} g(\zeta) \Gamma^{-\frac{1}{2}} d \zeta+\frac{i}{\pi} \int_{z=i r}^{s+i r} g(\zeta) L\left[H^{(0)}\right] \Gamma^{-\frac{1}{2}} d \zeta \equiv 0 .
\end{aligned}
$$

It also follows from Lemma 1 that $\Phi(0, z, g)=g(z)$ and $\Phi_{r}(0, z ; g) \equiv 0$. We have thus given an explicit construction for the function in Theorem 2 as

$$
u(r, z)=\Phi(r, z ; g) \text {. }
$$

We now return to the proof of Lemma 2. First the new variables $\varrho=r^{2}$, $\tau=(z-\zeta)^{2}, \quad E^{(n)}(\varrho, \tau, \zeta)=H^{(n)}(\sqrt{\varrho}, \zeta+\sqrt{\tau}, \zeta) \quad$ and $Q(\varrho, \tau, \zeta)=q(\sqrt{\varrho}, \zeta+\sqrt{\tau})$ are introduced. Equation (3.14) then becomes

$$
8\left(n-\frac{1}{2}\right)\left[\varrho E_{\varrho}^{(n)}+\tau E_{\tau}^{(n)}+n E^{(n)} / 2\right]=M\left[E^{(n-1)}\right]
$$

where

$$
M\left[E^{(n-1)}\right]=4 \varrho E_{\varrho \varrho}^{(n-1)}+4 E_{\varrho}^{(n-1)}+4 \tau E_{\tau \tau}^{(n-1)}+2 E_{\tau}^{(n-1)}+Q E^{(n-1)} .
$$

Consider now the "straight lines" $L_{a b}$ given parametrically by $\varrho=a \sigma, \tau=b \sigma$ with $a^{2}+b^{2}=1, a$ and $b \geqq 0$ and $\sigma$ is a complex variable. On $L_{a b}$

$$
\varrho E_{\varrho}^{(n)}+\tau E_{\tau}^{(n)}+\frac{n}{2} E^{(n)}=\sigma \frac{d E^{(n)}}{d \sigma}+\frac{n}{2} E^{(n)}=\sigma^{1-\frac{n}{2}} \frac{d}{d \sigma} \sigma^{\frac{n}{2}} E^{(n)} .
$$

Hence along $L_{a b}$, equation (3.16) becomes an ordinary differential equation for $E^{(n)}$ once we are given $E^{(n-1)}$. The requirement that $E^{(n)}$ remains finite as $\varrho \rightarrow 0$ yields

$$
\begin{aligned}
\sigma^{\frac{n}{2}} E^{(n)}= & -\frac{1}{8\left(n-\frac{1}{2}\right)} \int_{0}^{\sigma} \sigma^{\frac{n}{2}-1}\left\{4 a \sigma E_{\varrho e}^{(n-1)}+4 E_{e}^{(n-1)}+\right. \\
& \left.+4 b \sigma E_{\tau \tau}^{(n-1)}+2 E_{\tau}^{(n-1)}+Q E^{(n-1)}\right\} d \sigma .
\end{aligned}
$$

We now propose to find a majorizing problem for the functions defined by (3.17) so that we may investigate the convergence of the series which defines $S(r, z, \zeta)$. Let us suppose that the points $(r, z)$ and $(\xi, \eta)$ where $\zeta=\xi+i \eta$ are in a closed bounded domain. Then there exists a constant $K$ such that $|r| \leqq K$, $|z| \leqq|K| / 2$ and $|\zeta| \leqq K / 2$ and hence $|\varrho| \leqq K^{2}$ and $|\tau| \leqq K^{2}$. Now we recall that $q(r, z)$ is an entire function of $r^{2}$ and $z$. Hence, given any positive constant $A$, we can find a sufficiently large positive constant $B$ such that *

$$
Q(\varrho, \tau, \zeta)=q(\sqrt{\varrho}, \zeta+\sqrt{\tau}) \ll B\left[\left(1-\frac{\zeta}{A}\right)\left(1-\frac{\sqrt{\varrho}}{A}\right)\left(1-\frac{\sqrt{\tau}}{A}\right)\right]^{-1}
$$

\footnotetext{
* Given the infinite series $\sum a_{m n p} x_{1}^{m} x_{2}^{n} x_{3}^{p}$ and $\left|a_{m n p}\right| \leqq \tilde{a}_{m n p}$ where $\tilde{a}_{m n p} \geqq 0$ for all $m, n, p \geqq 0$. We write

$$
\sum a_{m n p} x_{1}^{m} x_{2}^{n} x_{3}^{p} \ll \sum \tilde{a}_{m n p} x_{1}^{n} x_{2}^{n} x_{3}^{p}
$$

and say that the second series dominates the first one. This dominance is also preserved under differentiation and integration $[8$, p. 289].
} 
for $|\zeta|,|\sqrt{\varrho}|$ and $|\sqrt{\tau}|<A$. Given the constant $K$, we choose $A$ such that $K / A<1$, and we have from (3.18) that

But

$$
Q \ll 2 B\left[\left(1-\frac{\sqrt{\varrho}}{A}\right)\left(1-\frac{\sqrt{\bar{r}}}{A}\right)\right]^{-1}
$$

and

$$
\left(1-\frac{\sqrt{\tau}}{A}\right)^{-1}=\left(1+\frac{\sqrt{\tau}}{A}\right)\left(1-\frac{\tau}{A^{2}}\right)^{-1} \ll 2\left(1-\frac{\tau}{A^{2}}\right)^{-1}
$$

and hence

$$
\left(1-\frac{V^{\prime} \bar{\varrho}}{A}\right)^{-1}=\left(1+\frac{\sqrt{\varrho}}{A}\right)\left(1-\frac{\varrho}{A^{2}}\right)^{-1} \ll 2\left(1-\frac{\varrho}{A^{2}}\right)^{-1}
$$

$$
Q \ll 8 B\left[\left(1-\frac{\varrho}{A^{2}}\right)\left(1-\frac{\tau}{A^{2}}\right)\right]^{-1} \ll 8 B\left[1-\frac{\varrho+\tau}{A^{2}}\right]^{-1} .
$$

On the "straight lines" $\varrho=a \sigma, \tau=b \sigma$ we have then

$$
Q \ll 8 B\left[1-\frac{\sigma}{\delta}\right]^{-1}
$$

where $\delta=A^{2} /(a+b)$ and $\sigma=\left[\varrho^{2}+\tau^{2}\right]^{\frac{1}{2}}$. Having found a dominant for $Q$, we define the functions $F^{(n)}(\varrho, \tau)$ by the recursion relation

$$
\begin{aligned}
\sigma^{\frac{n}{2}} F^{(n)}= & \frac{1}{8\left(n-\frac{1}{2}\right)} \int_{0}^{\sigma} \sigma^{\frac{n}{2}-1}\left\{4 a \sigma F_{\varrho \varrho}^{(n-1)}+4 F_{\varrho}^{(n-1)}++\right. \\
& \left.+4 b \sigma F_{\tau \tau}^{(n-1)}+2 F_{\tau}^{(n-1)}+8 B\left(1-\frac{\sigma}{\delta}\right)^{-1} F^{(n-1)}\right\} d \sigma .
\end{aligned}
$$

We now choose $E^{(0)}=F^{(0)}=1$, from which we see immediately that $E^{(\mathbf{1})} \ll F^{(1)}$ since $0 \leqq a \leqq 1,0 \leqq b \leqq 1$ and $a^{2}+b^{2}=1$. Upon repeating this process for $n \geqq 1$ and using the properties of the dominants, we find that $E^{(n)} \ll F^{(n)}$ where the arguments in (3.19) are still $\varrho=a \sigma$ and $\tau=b \sigma$.

We now assert that for $n$ sufficiently large

and

$$
F^{(n)}(\varrho, \tau) \ll \frac{C_{n}}{\delta^{n}}\left(1-\frac{\sigma}{\delta}\right)^{-n}
$$

$$
\lim _{n \rightarrow \infty} \frac{C_{n}}{C_{n-1}}=1
$$

We prove this by induction. First it is noted that $(3.20)$ is trivially true for $n=0$. Let us assume it is true for $n-1$. Then on $\varrho=a \sigma, \tau=b \sigma$ with $a^{2}+b^{2}=1$, we have

where

$$
\begin{aligned}
4 a \sigma F_{\varrho \varrho}^{(n-1)} & +4 F_{\varrho}^{(n-1)}+4 b \sigma F_{\tau \tau}^{(n-1)}+2 F_{t}^{(n-1)}+8 B\left(1-\frac{\sigma}{\delta}\right)^{-1} F^{(n-1)} \\
& \ll 4 n(n-1) C_{n-1}\left(1-\frac{\sigma}{\delta}\right)^{-n-1} \delta^{-n-1}\left[\left(a^{3}+b^{3}\right) \sigma+\alpha_{n}(\sigma)\right]
\end{aligned}
$$

$$
\alpha_{n}(\sigma)=\left(1-\frac{\sigma}{\delta}\right) \frac{\delta}{n}\left[a b^{2}+b a^{2}+a+\frac{b}{2}+2 B \frac{\delta^{2}}{n-i}\right] .
$$

Now since $\delta$ is fixed, an $n_{0}$ can be chosen sufficiently large that

$$
\frac{1}{n}\left\{a b^{2}+b a^{2}+a+\frac{b}{2}+2 B \frac{\delta^{2}}{n-1}\right\}<1 \text { for } n \geqq n_{0} .
$$


Since $a^{3}+b^{3} \leqq 1$, we have from (3.19)

We also have that

$$
\sigma^{\frac{n}{2}} F^{(n)} \ll \frac{n(n-1) C_{n-1}}{2\left(n-\frac{1}{2}\right) \delta^{n+1}}\left\{\int_{0}^{\sigma} \frac{\sigma^{\frac{n}{2}} d \sigma}{\left(1-\frac{\sigma}{\delta}\right)^{n+1}}+\int_{0}^{\sigma} \frac{\delta \sigma^{\frac{n}{2}-1} d \sigma}{\left(1-\frac{\sigma}{\delta}\right)^{n}}\right\} .
$$

$$
\frac{2}{n+2} \cdot \frac{d}{d \sigma} \frac{\sigma^{\frac{n}{2}+1}}{\left(1-\frac{\sigma}{\delta}\right)^{n}}=\frac{\sigma^{\frac{n}{2}}}{\left(1-\frac{\sigma}{\delta}\right)^{n+1}}\left\{1+\frac{n-2}{n+2} \frac{\sigma}{\delta}\right\} \gg \frac{\sigma^{\frac{n}{2}}}{\left(1-\frac{\sigma}{\delta}\right)^{n+1}}
$$

and

Hence

$$
\frac{2}{n} \frac{d}{d \sigma} \frac{\sigma^{\frac{n}{2}}}{\left(1-\frac{\sigma}{\delta}\right)^{n-1}}=\frac{\sigma^{\frac{n}{2}-1}}{\left(1-\frac{\sigma}{\delta}\right)^{n}}\left\{1+\frac{n-2}{n} \frac{\sigma}{\delta}\right\} \gg \frac{\sigma^{\frac{n}{2}-1}}{\left(1-\frac{\sigma}{\delta}\right)^{n}} .
$$

$$
F^{(n)} \ll \frac{(n-1) C_{n-1}\left(1-\frac{\sigma}{\delta}\right)^{-n}}{\left(n-\frac{1}{2}\right) \delta^{n}},
$$

and this is the form (3.20) with

$$
\frac{C_{n}}{C_{n-1}}=\frac{n-1}{n-\frac{1}{2}}
$$

so that (3.21) is also satisfied. It is now a simple task to complete the proof of Lemma 2. We have shown that

$$
H^{(n)}(\sqrt{\varrho}, \zeta+\sqrt{\tau}, \zeta)=E^{(n)}(\varrho, \tau, \zeta) \ll C_{n}\left(1-\frac{\sigma}{\delta}\right)^{-n} \delta^{-n}
$$

Now the series

is majorized by the series

$$
\sum_{n=0}^{\infty} H^{(n)} \Gamma^{n}
$$

$$
\sum_{n=0}^{\infty} C_{n}\left(1-\frac{\sigma}{\delta}\right)^{-n} \delta^{-n} \Gamma^{n}=\sum_{n=0}^{\infty} C_{n}\left(1-\frac{\sigma}{\delta}\right)^{-n} \delta^{-n}(a+b)^{n} \cdot \sigma^{n},
$$

and this will converge uniformly and absolutely when

$$
\frac{(a+b)|\sigma|}{|\delta-\sigma|}<1
$$

Now we return to the series

$$
\sum_{n=0}^{\infty} H^{(n)} \Gamma^{n}
$$

whose convergence we wish to study. Let us first observe that

$$
|\sigma|=\left|\sqrt{\varrho^{2}+\tau^{2}}\right|<|\varrho|+|\tau| .
$$

Further for $|r|^{2}=|\varrho| \leqq K^{2},|z| \leqq K / 2,|\zeta| \leqq K / 2,|\tau| \leqq K^{2}$, and hence $|\Gamma| \leqq$ $|\varrho|+|\tau| \leqq 2 K^{2}$ so that

$$
\left|\sum_{n=0}^{\infty} H^{(n)} \Gamma^{n}\right| \leqq \sum_{n=0}^{\infty}\left|C_{n}\right| \delta^{-n}\left(2 K^{2}\right)^{n}\left(1-\frac{|\varrho|+|\tau|}{\delta}\right)^{-n}
$$


Since

we have.

$$
\left(1-\frac{|\varrho|+|\tau|}{\delta}\right)^{-1} \leqq\left(1-\frac{2 K^{2}}{\delta}\right)^{-1}
$$

$$
\left|\sum_{n=0}^{\infty} H^{(n)} \Gamma^{n}\right| \leqq \sum_{n=0}^{\infty}\left|C_{n}\right| \delta^{-n}\left(2 K^{2}\right)^{n}\left(1-\frac{2 K^{2}}{\delta}\right)^{-n}
$$

It is also to be noted that since $\delta=A^{2} /(a+b), \dot{a}^{2}+b^{2}=1$ and $a$ and $b$ are positive, $1 / \delta<2 / A^{2}$ and $1 / \delta \geqq 1 / A^{2}$. Hence we have finally

$$
\left|\sum_{n=0}^{\infty} H^{(n)} \Gamma^{n}\right| \leqq \sum_{n=0}^{\infty}\left|C_{n}\right|\left(\frac{4 K^{2}}{A^{2}}\right)^{n}\left(1-\frac{2 K^{2}}{A^{2}}\right)^{-n},
$$

and this series converges for $6 K^{2}<A^{2}{ }^{\star}$ This completes the proof of Lemma 2 .

There is a modification of the representation (3.1) which can be obtained from the following lemma.

Lemma 3. The functions $H^{(n)}$ defined by the recursion relation (3.14) satisfy

$$
H^{(n)}(r, z, \bar{\zeta})=\overline{H^{(n)}(r, z, \zeta)} .
$$

We prove this by induction. Since $H^{(0)} \equiv 1,(3.23)$ is true for $n=0$. Let us assume (3.23) is true for $n-1$ and consider the recursion formula (3.14) for $\bar{\zeta}$. Upon putting $\psi^{(n)}(r, z, \zeta)=\overline{H^{(n)}(r, z, \bar{\zeta})}$, we obtain

$$
4\left[n-\frac{1}{2}\right]\left[r \psi_{r}^{(n)}+(z-\bar{\zeta}) \psi_{r}^{(n)}+n \psi^{(n)}\right]=-L\left[\psi^{(n-1)}\right],
$$

and the conjugate of this is

$$
4\left[n-\frac{1}{2}\right]\left[r \bar{\psi}_{r}^{(n)}+(z-\zeta) \bar{\psi}_{r}^{(n)}+n \bar{\psi}^{(n)}\right]=-L\left[\bar{\psi}^{(n-1)}\right] .
$$

The induction hypothesis states that $\bar{\psi}^{(n-1)}=H^{(n-1)}$, so that if we subtract equation (3.24) from (3.14), we get

$$
m_{n}\left[\bar{\psi}^{(n)}-H^{(n)}\right] \equiv 0
$$

But we have just seen that this implies $\bar{\psi}^{(n)} \equiv H^{(n)}$, and this is (3.23). For the function $S(r, z, \zeta)$ of Lemma 2 we have

$$
\begin{aligned}
S(r, z, \bar{\zeta})\left\{r^{2}+(z-\bar{\zeta})^{2}\right\}^{-\frac{1}{2}} & =\sum_{n=0}^{\infty} H^{(x)}(r, z, \zeta)\left\{r^{2}+(z-\bar{\zeta})^{2}\right\}^{n-1} \\
& =\sum_{n=0}^{\infty} \overline{H^{(n)}(r, z, \zeta)}\left\{\overline{r^{2}+(z-\bar{\zeta})^{2}}\right\}^{n-1}
\end{aligned}
$$

Let us suppose now that the region $R$ of Theorem 2 has the special property that if it contains the point $\zeta=z+i r$, it contairs the segment $\zeta=z+i \varrho$, $-r \leqq \varrho \leqq r$. Such a region will be called simple. If we now choose this segment as the path of integration in the representation (3.15), we find that

$$
u(r, z)=\frac{1}{\pi} \int_{-r}^{r} S(r, z, z+i \varrho) g(z+i \varrho)\left(r^{2}-\varrho^{2}\right)^{-1} d \varrho
$$

\footnotetext{
* With little effort the constant 6 may be reduced to $2(1+\sqrt{2})$, but the main issue is that $K^{2} / A^{2}$ be less than unity.
} 
But $g(\zeta)=u(0, \zeta)$ and hence is real for $\zeta$ real, that is, $g(\bar{\zeta})=\overline{g(\zeta)}$. Accordingly, we may write (3.26) as

$$
u(r, z)=\frac{2}{\pi} \int_{0}^{r}\left(r^{2}-\varrho^{2}\right)^{-\frac{1}{2}} \operatorname{Re}[S(r, z, z+i \varrho) g(z+i \varrho)] d \varrho .
$$

We shall see presently that (3.27) will be useful in some special cases.

There is another property of the functions $H^{(n)}$ and the series $S$ formed from them which we wish to point out. With the function $S$ defined as in Lemma 2 and observing the mode of construction of the $H^{(n)}$, we have the following lemma.

Lemma 4. If $\zeta$ is real and the operator $L$ is defined as in (2.1), the function

$$
V(r, z, \zeta)=S(r, z, \zeta)\left[r^{2}+(z-\zeta)^{2}\right]^{-\frac{1}{2}}
$$

will satisfy the following conditions:

(i) $L(V)=0$ for $(r, z) \neq(0, \zeta)$

and

(ii) $V_{r}(0, z, \zeta)=0$ for $z \neq \zeta$.

The condition (ii) follows from the requirement that $V$ is a RSF in any region which intersects $r=0$ and does not contain $z=\zeta$.

\section{A Regularity Property}

In Theorem 2 we demonstrated that for a real analytic function $g(z)$ on a segment $\lambda$ of the $z$-axis to be the boundary value of a RSF in a region $R$, it was sufficient that the extension $g(\zeta)$ to complex $\zeta$ be analytic in $R$. We now prove in this section that this condition is also necessary. We have here

Theorem 3. Suppose $u(r, z)$ is a $R S F$ in a region $R$ with $\lambda=R \cap(r=0)$. Then the function $g(\zeta)$ which is the extension to complex $\zeta=z+i r$ of $u(0, z)$ on $\lambda$ is analytic in $R$.

The proof of this theorem follows that of ERDÉLYI [2] for the special case of equation (1.1). From Lemma 4 we have at our disposal a solution $V(r, z, \zeta)$ of equation (2.1) with a singular point on the axis of symmetry. Let $\gamma_{\varepsilon}$ denote a semi-circle of radius $\varepsilon$ about the point $(0, \zeta)$. Since $S(r, z, \zeta)$ is an analytic function for $(r, z)$ in the neighborhood of $(0, \zeta)$, we have

$$
\frac{\partial}{\partial n} V(r, z, \zeta)=S \varepsilon^{-2}+O\left(\varepsilon^{-1}\right)
$$

where $(r, z)$ is on $\gamma_{\varepsilon}$ and $\vec{n}$ denotes the normal on $\gamma_{s}$ directed toward $(0, \zeta)$. Now we apply Green's theorem [2] to obtain

$$
\int_{C} r\left[u \frac{\partial v}{\partial n}-v \frac{\partial u}{\partial n}\right] d S=\iint_{D} r\{u e[v]-v e[u]\} d r d z
$$

which is valid for any two functions $u$ and $v$ of class $C^{(2)}$ in a domain $D$ bounded by a curve $C$ which is sufficiently smooth. The normal $\vec{n}$ denotes the exterior normal to $D$, and the operator $e$ is defined by

$$
e[f]=f_{r r}+r^{-1} f_{r}+f_{s x} \text {. }
$$


Let $R$ be the region of Theorem 3. By definition $R$ is symmetric with respect to $r=0$. Let $C_{\varepsilon}$ denote $R \cap(r=0)$ minus the segment $r=0,|z-\zeta| \leqq \varepsilon$ and $C_{1}$ the complement of $C_{s}+\gamma_{s}$ with respect to $C$, the path $C$ having been closed in the half plane $r>0$. Now we apply* (4.2) with $u$ the RSF of Theorem 3, $v=V(r, z, \zeta)$ and $D$ the domain bounded by $C_{1}, C_{\varepsilon}$ and $\gamma_{s}$. Since $u$ is a RSF, it follows that $u_{n} \equiv 0$ on $C_{\varepsilon}$, and by Lemma 4 we also have that $V_{n}=0$ on $C_{\varepsilon}$. If we take the limit $\varepsilon \rightarrow 0$ and use (4.1), we obtain

$$
u(0, \zeta)=\pi^{-1} \int_{C_{1}} r\left[u \frac{\partial V}{\partial n}-V \frac{\partial u}{\partial n}\right] d s .
$$

From Lemma 2 we have that $V$ is an analytic function of $(r, z, \zeta)$ as long as $(r, z) \neq(0, \zeta)$. It is thus permissible to allow $\zeta$ to become complex, and $u(0, \zeta)$ will be an analytic function of $\zeta$ as long as

$$
r^{2}+(z-\zeta)^{2} \neq 0, \quad(r, z) \in C_{1},
$$

that is, for $\zeta \neq z \pm i r,(r, z) \in C_{1}$. Hence as long as $\zeta$ is interior to the region bounded by $C$ and its image with respect to $r=0, u(0, \zeta)$ is an analytic function of $\zeta$.

\section{Some Special Cases}

The representations we have developed simplify considerably in the special case that $q$ is independent of $z$. Let us suppose that $q(r, z)=f(r)$, where now $f(r)$ is an analytic function of $r^{2}$. From equation (3.17) we obtain the following result immediately.

Lemma 5. If $q(r, z)$ is independent of $z$, the functions $H^{(n)}$ satisfy

$$
H^{(n)}(r, z, \zeta)=h^{(n)}(r), \quad \cdot H^{(0)}(r) \equiv 1
$$

and $h^{(n)}(r)$ is real for real $r$.

Let us now suppose that the region $R$ is simple. Then (3.26) applies, and from Lemma 5 we have that $S(r, z, \zeta) \Gamma^{n-1}$ is real for $\zeta=z+i \varrho$. For $q$ independent of $z$, we write $S(r, z, z+i \varrho)=G(r, \varrho)$, and (3.26) becomes

$$
u(r, z)=\frac{2}{\pi} \int_{0}^{r}\left(r^{2}-\varrho^{2}\right)^{-\frac{1}{2}} G(r, \varrho) \operatorname{Re} f(z+i \varrho) d \varrho .
$$

The function $\varphi(\varrho, z)=\operatorname{Re} f(z+i \varrho)$ is a harmonic function in the two variables $z$ and $\varrho$, and it is regular in the same region $R$ as $f(\zeta)=u(0, \zeta)$. We further note that the condition $f(\zeta)=\overline{f(\bar{\zeta})}$ implies that $\varphi(\varrho, z)$ is a harmonic function which is symmetric in $r$, that is, $\varphi(\varrho, z)=\varphi(-\varrho, z)$.

There is another type of inversion formula by means of which we can recover the harmonic function $\varphi(\varrho, z)$ from the RSF $u(r, z)$. We introduce the variables

$$
\alpha=r^{2}, \quad \beta=\varrho^{2}, \quad \Phi(\beta, z)=\varphi(\sqrt{\beta}, \dot{z}) / \sqrt{\beta}, \quad u(\alpha, z)=u(\sqrt{\alpha}, z)
$$

$\star$ We have assumed here that the RSF $u$ of Theorem 3 is of class $C^{(2)}$ in $R$. If it is not, we apply the same method to a slightly smaller region $R^{\prime}$ contained in $R$. Then the analyticity of $u$ in $R$ shows that the hypotheses of (4.2) are satisfied. 
in (5.1). Then for each fixed $z,(5.1)$ becomes

$$
u(\alpha, z)=\pi^{-1} \int_{0}^{\alpha}(\alpha-\beta)^{-1} G(\sqrt{\alpha}, \sqrt{\beta}) \Phi(\beta, z) d \beta .
$$

Since the $H^{(n)}$ are analytic functions of $\gamma^{2}$ so are the $h^{(n)}$. Hence $G(\sqrt{\alpha}, \sqrt{\beta})$ is an analytic function of $\alpha$ and $\beta$ and indeed

$$
G(\sqrt{\alpha}, \sqrt{ } \beta)=\sum_{k=0}^{\infty} g^{(n)}(\alpha-\beta)^{n}, \quad g(0) \equiv 1
$$

For each fixed $z,(5.2)$ is then a Volterra integral equation for $\Phi(\beta, z)$, the range of $\beta$ being $0<\beta<b_{1}(z)$ where $r=b_{1}(z)$ is the equation which defines the boundary of $R$.

Equation (5.2) may be converted into an integral equation of the second kind by means of the following lemma.

Lemma 6. The integral operator $T$ given by

has the unique inverse

$$
T(h)=\frac{1}{\pi} \int_{0}^{\alpha}(\alpha-\beta)^{-\frac{1}{2}} h(\beta) d \beta=g(\alpha)
$$

$$
T^{-1}\left(\delta^{\prime}\right)=\frac{d}{d \alpha} \int_{0}^{\alpha}(\alpha-\beta)^{-\frac{1}{2}} g(\beta) d \beta=h(\alpha)
$$

for all functions $g(\beta)$ which are continuous on $0 \leqq \beta \leqq b$.

From Lemma 6 and equation (5.3) we may now rewrite (5.2) as

$$
\Phi(\beta, z)+\frac{1}{\pi} T^{-1}\left\{\sum_{n=1}^{\infty} \int_{0}^{\alpha}(\alpha-\gamma)^{n-1} g^{(n)}(\gamma) \Phi(\gamma, z) d \gamma\right\}=T^{-1} U
$$

which is an integral equation of the second kind.

We now specialize our results further by supposing that $q(r, z)$ is a constant $\mu$. It is easily verified by induction that (3.26) yields in this case

so that

$$
H^{(x)}=\frac{(-)^{n} \mu^{n}}{(2 n) !}
$$

$$
\begin{aligned}
S(r, z, \zeta) \Gamma^{-1} & =\sum_{n=0}^{\infty} \frac{(-)^{n} \mu^{n}}{(2 n) !}\left[r^{2}+(z-\zeta)^{2}\right]^{n-1} \\
& =\left[r^{2}+(z-\zeta)^{2}\right]^{-\frac{1}{2}} \cos \left\{\mu\left[r^{2}+(z-\zeta)^{2}\right]\right\}^{\frac{1}{2}}
\end{aligned}
$$

In this case (3.14) and (3.15) yield the form which HENRICI gives in [3] once a minor change of variables is made, and, if $\mu=0$, they yield the Laplace integral (1.2). In this special case, the inversion formula for equation $(5: 2)$ can be obtained explicitly, and indeed we have

$$
p(r, z)=\frac{1}{2} \frac{d}{d r} \int_{0}^{r}\left(r^{2}-\varrho^{2}\right)^{-1} \cosh \left\{\left[\mu\left(r^{2}-\varrho^{2}\right)\right]\right\}^{-1} u(\varrho, z) d \varrho .
$$

This result has been very useful in the study of the diffraction of sound waves by a circular disk [7].

This work was supported in part by the Air Force Office of Scientific Research. 


\section{References}

[1] Weinstein, A.: Generalized axially symmetric potential theory. Bull. Amer. Math. Soc. 59, No. 1, 20-38 (1953).

[2] ERDÉlyi, A.: Singularities on generalized axially symmetric potentials. Comm. on Pure and App. Math. 9, No. 3, 403-414 (1956).

[3] Henrici, P.: Zur Funktionentheorie der Wellengleichung. Comm. Math. Helv. 27, 235-293 (1953).

[4] Henrici, P.: A survey of I. N. Vekua's theory of partial differential equations with analytic coefficients. ZAMP 8, 169-203 (1957).

[5] Henrici, P.: Axially-symmetric potential functions. Proceedings, Conference on boundary problems in partial differential equations. U. S. Army Mathematics Research Center, Univ. of Wisconsin 1960.

[6] Heins, A. E., \& R. C. MACCAMY: On mixed boundary-value problems for axiallysymmetric potentials. J. of Math. Anal. and App. 1, No. 3, 331-333 (1960).

[7] Heins, A. E., \& R. C. MacCAmy: On the scattering of waves by a disk. Z. Angew. Math. und Phys. 11, No. 4, 249-264 (1960).

[8] Bergman, S., \& M. Schiffer: Kernel Functions and Differential Equations. New York: Academic Press 1953.

[9] Diaz, J. B., \& G. S. S. Ludford: On the singular Cauchy problem for a generalization of the Euler-Poisson-Darboux equation in two space variables. Annali di Math. (38) Series 4, 33-50 (1955).

[10] Darboux, G.: Leçons sur la théorie générale des surfaces, Vol. 2. Paris 1915.

University of Michigan

Ann Arbor, Michigan

and

Carnegie Institute of Technology

Pittsburgh, Pennsylvania

(Received April 16, 1963) 